\title{
TEM and EDX of Metallic Nanoparticles in e-Cigarette Aerosol
}

Hendrik O. Colijn ${ }^{1}$ and Vladimir Mikheev ${ }^{2}$

${ }^{1 .}$ Center for Microscopy and Analysis, The Ohio State University, Columbus, $\mathrm{OH}$.

2. Battelle Memorial Institute, Columbus, $\mathrm{OH}$.

E-cigarettes have recently become quite popular [1] and have a reputation of being less harmful than regular cigarettes [2]. Few analyses have been done on the aerosols/vapors emitted from these devices but there is some evidence that the emitted aerosol contains metallic nanoparticles. It has been difficult to characterize these particles [3].

Aerosol generated by blu e-cigarettes was collected on aluminum foils using an electric low pressure impactor (ELPI, Fig 1.). To examine the collected particles, it was first necessary to remove the majority of the condensed glycerol and propylene glycol by heating the sample over an incandescent lamp overnight. After taping the filter onto a slide, thin cellulose acetate replicating tape softened with acetone was placed on the foil and gently pressed onto the surface. Once dry, the replicating tape was peeled off and was taped, particle side up, to a new slide. The replica was carbon coated with $30-40 \mathrm{~nm}$ carbon using a high-vacuum carbon-thread coater equipped with a quartz crystal thickness monitor. A 1-2mm section of the carbon-coated replica was cut from an area near the center of the filter and transferred to a TEM grid with the carbon side up. The grid with sample was placed in a petri dish with on several sheets of filter paper. The filter paper was then saturated with acetone to remove the replica material. After several rinses, the grids were checked in the TEM. Many of the grids showed significant carbonaceous buildup under the e-beam. These grids were then treated on both sides with a low-power ozone-generating UV lamp $(185 \& 254 \mathrm{~nm})$ to reduce the contamination buildup to acceptable levels. There appears to be a greater concentration of the nanoparticles within the condensate on the foils, so TEM grids taken from different areas of the foil may have a large variation in nanoparticle concentration.

Examination of the samples was performed in an FEI Tecnai F20 TEM/STEM equipped with an EDAX $30 \mathrm{~mm}^{2}$ windowless EDX detector. A typical TEM image of the nano-particles is in Figure 2. Figure 3 is an HAADF STEM image of nanoparticles with the area of an EDX map marked. The EDX map indicates the presence of copper in the nanoparticles while their spectra shows only copper and no other metal above background. Spectra from areas adjacent to the particles show no metal presence.

We have developed a method for examining metallic nanoparticles in e-cigarette aerosol and confirmed the presence of $10-20 \mathrm{~nm}$ copper nanoparticles. These inhaled nanoparticles have the potential to reach sensitive target sites in the body and have been observed translocating along axons and neuron dendrites. Copper has potential cytotoxicity and in the form of nanoparticles may provoke oxidative stress in the affected cells [4].

References:

[1] Delnevo CD et al, Nicotine \& Tobacco Research 18 (2015), p. 715.

[2] Farsalinos KE, Polosa R., Therapeutic Advances in Drug Safety 5 (2014), p. 67.

[3] Williams M et al, PLoS One 8 (2013), p. e57987. 
[4] The authors acknowledge support from The Ohio State University and the Ohio Third Frontier Research Scholar program and from grant number P50CA180523 from the National Cancer Institute and FDA Center for Tobacco Products (CTP).
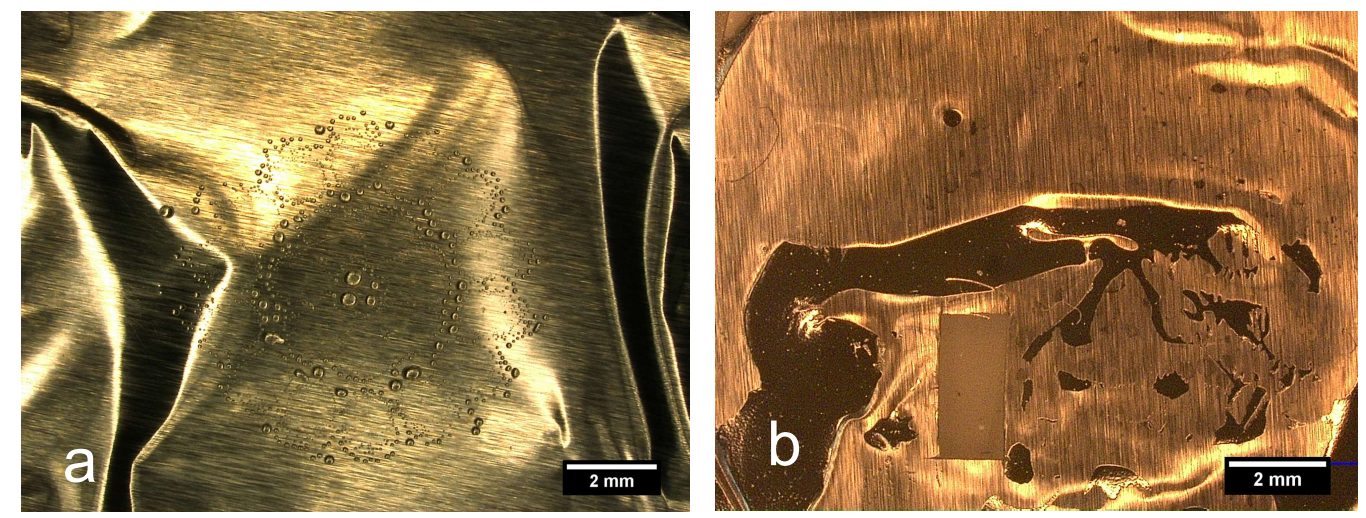

Figure 1. (a) Aluminum impact foil with vapor condensate. (b) carbon replica

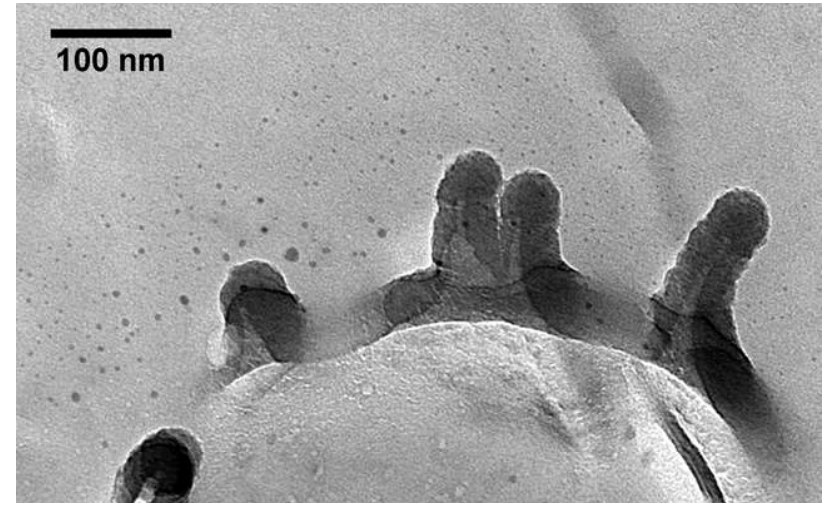

Figure 2. TEM image of filter deposit with metallic nano-particles.
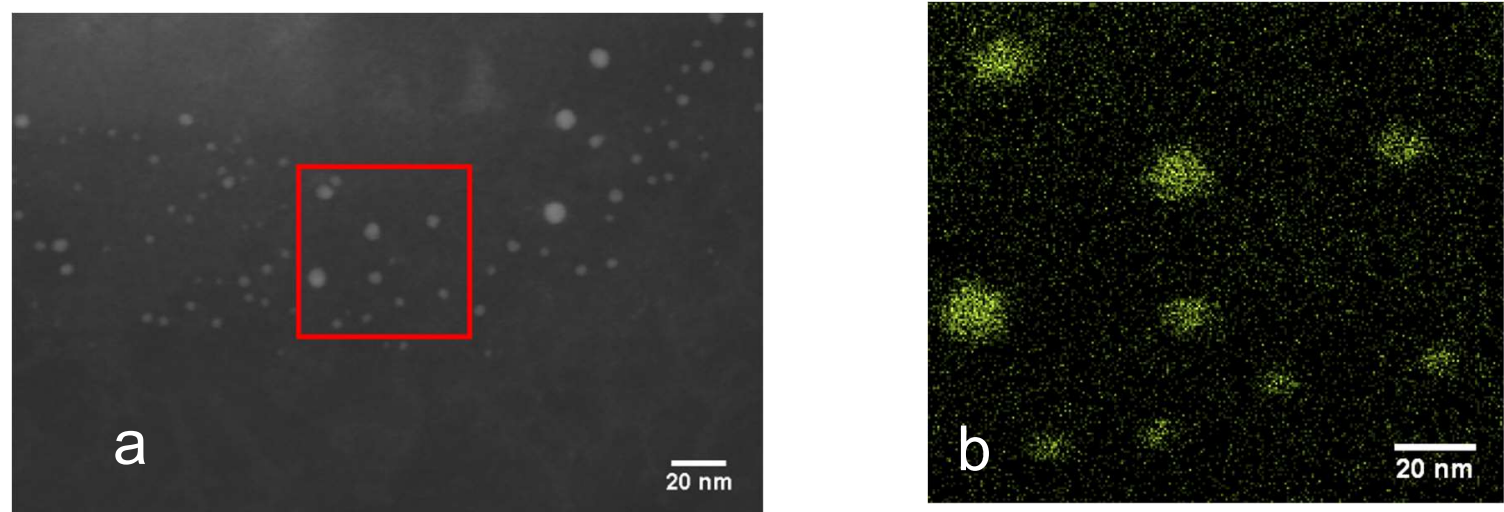

Figure 3. (a) HAADF STEM image of indicating area of EDX map. (b) Copper EDX map of nanoparticles. 